ARTICLE

https://doi.org/10.1038/s41467-020-16136-5

\title{
Single-step manufacturing of hierarchical dielectric metalens in the visible
}

\author{
Gwanho Yoon (1) 1,5, Kwan Kim (1D 2,5, Daihong Huh², Heon Lee (D) ${ }^{2 凶}$ \& Junsuk Rho (D) 1,3,4凶
}

Metalenses have shown a number of promising functionalities that are comparable with conventional refractive lenses. However, current metalenses are still far from commercialization due to the formidable fabrication costs. Here, we demonstrate a low-cost dielectric metalens that works in the visible spectrum. The material of the metalens consists of a matrix-inclusion composite in which a hierarchy satisfies two requirements for the single-step fabrication; a high refractive index and a pattern-transfer capability. We use a UV-curable resin as a matrix to enable direct pattern replication by the composite, and titanium dioxide nanoparticles as inclusions to increase the refractive index of the composite. Therefore, such a dielectric metalens can be fabricated with a single step of UV nanoimprint lithography. An experimental demonstration of the nanoparticle composite-based metalens validates the feasibility of our approach and capability for future applications. Our method allows rapid replication of metalenses repeatedly and thereby provides an advance toward the use of metalenses on a commercial scale.

\footnotetext{
${ }^{1}$ Department of Mechanical Engineering, Pohang University of Science and Technology (POSTECH), Pohang 37673, Republic of Korea. ${ }^{2}$ Department of Materials Science and Engineering, Korea University, Seoul 02841, Republic of Korea. ${ }^{3}$ Department of Chemical Engineering, Pohang University of Science and Technology (POSTECH), Pohang 37673, Republic of Korea. ${ }^{4}$ National Institute of Nanomaterials Technology (NINT), Pohang 37673, Republic of Korea.

${ }^{5}$ These authors contributed equally: Gwanho Yoon, Kwan Kim. ${ }^{凶}$ email: heonlee@korea.ac.kr; jsrho@postech.ac.kr
} 
M etasurfaces that consist of ultrathin subwavelength antenna arrays drive a paradigm shift from bulky and heavy to compact and light optics ${ }^{1,2}$. The strong interaction between electromagnetic (EM) waves and antenna allows efficient light modulation at the subwavelength scale ${ }^{3}$, in contrast to typical refractive components that operate at the wavelength scale. Metasurfaces can control the amplitude, phase, polarization, and frequency of EM waves by adjusting the configuration of antennas, and can realize several promising applications, such as diffraction-limited imaging ${ }^{4,5}$, high-resolution holography ${ }^{6-8}$, optical cloaking 9 , and structural color printing ${ }^{10,11}$. Simultaneous control of more than one wave property is also feasible with a single antenna enabling multifunctional operations ${ }^{12,13}$. Furthermore, multilayered metasurfaces provide a great opportunity for functional integration ${ }^{14,15}$.

Metalenses designed to focus light have emerged as nextgeneration lenses. Spherical aberration can be perfectly removed in metalenses without trouble whereas conventional aspherical lenses require costly and time-consuming processes ${ }^{16}$. Early plasmonic metalenses suffered from low diffraction efficiencies ${ }^{17}$ that were drastically increased by using dielectric antennas that induce strong electric and magnetic resonances ${ }^{3}$. Geometric phase from anisotropic antennas realizes broadband operation with polarization dependence ${ }^{18,19}$, and is favorable for use in augmented reality devices ${ }^{20}$. Propagation phase from isotropic antennas enables the polarization-insensitive operation of metalenses ${ }^{21}$. Appropriate control of both the geometric and propagation phase of antennas can compensate for chromatic aberration of metalenses which is a characteristic of diffractive optical elements ${ }^{22,23}$. The chromatic aberration can also be eliminated by other methods such as transmission amplitude modulation $^{14}$ or algorithm-assisted optimization ${ }^{24}$. Dielectric metalenses have the potential to replace conventional bulky and heavy lenses, but fabrication limitations impede the commercialization of metalenses.

The fabrication of dielectric metalenses suffers from both high manufacturing costs and low throughput. Electron beam lithography (EBL) is widely used to fabricate metalenses because they require subwavelength structures of sizes smaller than the diffraction limit of photolithography. The wavelength of an electron beam is only a few nanometers, so EBL can define metalenses with high pattern fidelity but the extremely low throughput hinders large-scale fabrication ${ }^{25}$. Nanoimprint lithography (NIL) has been evaluated as a way to manufacture metasurfaces on large scale 26,27 . The fabrication of the master molds for NIL requires EBL, but the replication from the master mold is much faster than EBL. However, conventional NIL also requires secondary operations such as thin-film deposition and etching ${ }^{20}$, so the production of metalenses by NIL is not yet competitive with the production of commercial plastic lenses using injection molding $^{28}$. These secondary operations reduce the productivity and degrade the substrate compatibility of NIL. Hence, the commercialization of metalenses requires the development of a one-step fabrication method.

Here, a low-cost hierarchical dielectric metalens is demonstrated using a single step of NIL. The fundamental requirement is that the pattern transferred from the mold should itself work as a metalens. Metalens patterns can be transferred to ultraviolet (UV)-curable resin by NIL, but the refractive index $n$ of typical resin is too low to work as a meta-atom ${ }^{29}$. Some commercial hybrid inorganic-organic materials have high $n$ in the visible range, but they suffer from high shrinkage and limited film thickness ${ }^{30}$. Therefore, we develop a nanoparticle composite (NPC) consisting of dielectric-NP inclusion in a matrix of UVcurable resin, and such a hierarchy enables the realization of lowcost dielectric metalenses. Titanium dioxide $\left(\mathrm{TiO}_{2}\right) \mathrm{NPs}$ are used in this work. $\mathrm{TiO}_{2}$ has much higher $n$ than the matrix, so the effective $n$ of the NPC can be increased and controlled by the content of $\mathrm{TiO}_{2}$ NPs. The matrix of the NPC is hardened by UV illumination, so the NPC pattern can be directly transferred from the mold and work as a metalens without any secondary operations. The final metalens is designated for a plum pudding metalens (PPML) because its unit structure resembles a plum pudding. Experimental demonstration of the PPML verifies the feasibility of our approach yielding a focusing efficiency of $33 \%$ at a design wavelength $\lambda_{\mathrm{d}}=532 \mathrm{~nm}$, and the efficiency can be increased by further optimization of the structure configuration. The maximum aspect ratio of the final NPC structures can exceed 10:1 due to the high stiffness of the NPC after UV curing. Largescale fabrication is also feasible using large-sized master molds. Since intermediate soft molds are reusable, the PPML can be replicated repeatedly using only a single step of printing without any secondary operations. The absence of secondary operations allows the fabrication of the PPML on flexible substrates which are usually vulnerable to plasma damage. Therefore, our approach will provide real opportunities for practical applications of metalenses.

\section{Results}

Characteristics of $\mathrm{TiO}_{2}$ NPC. The PPML has a hierarchy of NPs embedded in a UV-curable resin (Fig. 1a). The material of the PPML must satisfy two requirements to realize low-cost metalens fabrication: a sufficiently-high $n$ to work as a meta-atom, and feasible transfer of the mold pattern. Therefore, we use the NPC as a material of the PPML to achieve those requirements. The NPC is a matrix-inclusion composite. The UV-curable resin is used as a matrix material to enable the replication of the mold pattern, and $\mathrm{TiO}_{2}$ NPs are embedded in the inclusions to increase the effective $n$ of the NPC. For the NPC to be a homogeneous effective medium, the $\mathrm{TiO}_{2} \mathrm{NPs}$ should be much smaller than the operating wavelength ${ }^{31}$, so $30 \mathrm{~nm}$ NPs are used in this work.

The $n$ of the NPC is characterized both theoretically and experimentally. The $n$ of the NPC is closely related to the filling fraction of the $\mathrm{TiO}_{2} \mathrm{NPs}$, so we characterize the optical properties of the NPC film by changing the ratio of $\mathrm{TiO}_{2} \mathrm{NPs}$. We prepare six kinds of NPCs from $0 \%$ to $100 \%$ of the $\mathrm{TiO}_{2}$ weight ratio (see Method), and they are spin-coated on a silicon substrate with the same spin-coating conditions (Fig. 1b). After UV curing, the final thickness of the NPC film decreases as the weight ratio increases due to the reduction in the amount of monomer. The coated NPC films are analyzed using ellipsometry, and the measured data is fitted using the Cauchy dispersion model which is expressed as

$$
n(\lambda)=A+\frac{B}{\lambda^{2}}+\frac{C}{\lambda^{4}}
$$

where $n$ is the refractive index, $\lambda$ is the wavelength, and $A, B, C$ are coefficients to match the model with the measured data. For example, the NPC film with $80 \%$ of the $\mathrm{TiO}_{2}$ weight ratio can be described by the model with coefficients of $A=1.853, B=$ 0.00864 , and $C=0.00414$ (Fig. 1c). The resulting mean square error between the model and the data is 23.7 which is low enough to validate that indeed the NPC acts as a homogeneous effective medium in the visible regime. The $n$ of the model is proportional to the $\mathrm{TiO}_{2}$ weight ratio, so the $n$ of the NPC can be controlled over a broad range by adjusting the weight ratio (Fig. 1d).

We also calculate the $n$ of the NPC by using the Maxwell-Garnett formula of the effective medium theory which yields the effective permittivity of the medium as ${ }^{32}$

$$
\varepsilon_{\text {eff }}=\varepsilon_{\mathrm{m}}+\beta\left(\varepsilon_{i}-\varepsilon_{\mathrm{m}}\right) \frac{3 \varepsilon_{\mathrm{m}}}{\varepsilon_{i}+2 \varepsilon_{\mathrm{m}}-\beta\left(\varepsilon_{i}-\varepsilon_{\mathrm{m}}\right)},
$$

where $\varepsilon_{\mathrm{m}}$ and $\varepsilon_{i}$ represent complex permittivity of the matrix and 

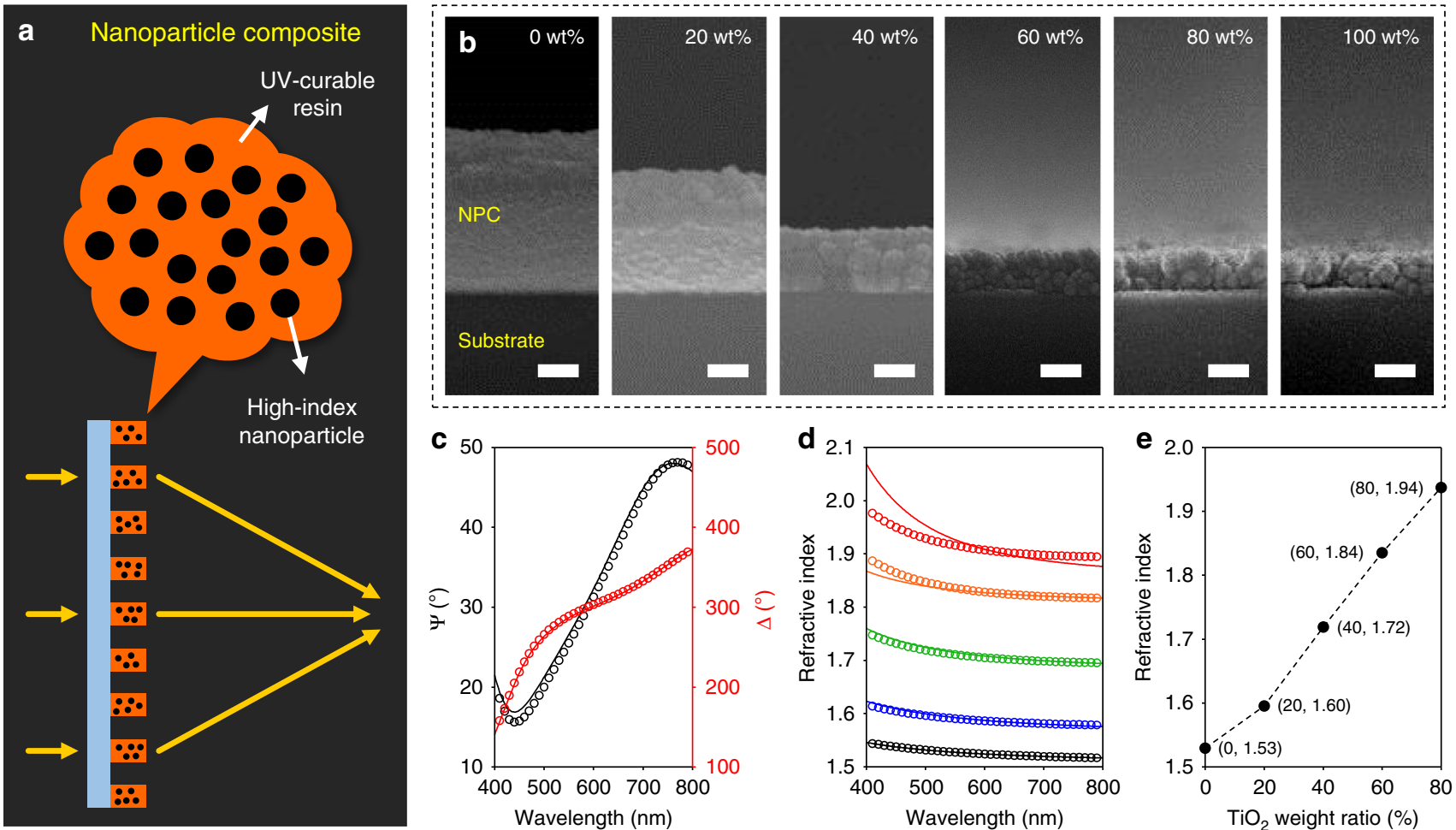

Fig. 1 Characterization of the nanoparticle composite. a Schematic of the plum pudding metalens (PPML). Yellow lines: light propagation direction through the PPML. The PPML can be fabricated with a single step of printing at low cost due to the compatibility of the nanoparticle composite (NPC). The refractive index of the NPC is high enough to realize metalenses, so the NPC can be directly used as a material of metalenses without any secondary operations such as thin-film deposition or etching. $\mathbf{b}$ Scanning electron micrographs of cross sections of the spin-coated NPC films with different weight ratios of titanium dioxide $\left(\mathrm{TiO}_{2}\right)$ nanoparticles. Spin-coating conditions are the same for all samples. The particle size is much smaller than the operating wavelength, so the NPC can work as a homogeneous effective medium. All scale bars: $150 \mathrm{~nm}$. c Ellipsometry analysis of the NPC film with $80 \%$ of the $\mathrm{TiO}_{2}$ weight ratio. The amplitude component is denoted by $\Psi$ while the phase difference is represented as $\Delta$. Real lines: theoretical prediction by the Cauchy dispersion model; circles: experimental measurements. d Comparison of the refractive index of the NPC films. Real lines: retrieved data from the ellipsometry measurement of each NPC film with different $\mathrm{TiO}_{2}$ weight ratio (black: 0\%; blue: 20\%; green: 40\%; orange: 60\%; red: $80 \%$ ); circles: calculated data by the Maxwell-Garnett formula with different volume fraction of $\mathrm{TiO}_{2}$ nanoparticles (Black: $0 \%$; blue: $7 \%$; green: $20 \%$; orange: $33 \%$; red: 41\%). e The measured refractive index variation of the NPC films by the weight ratio at the wavelength of $532 \mathrm{~nm}$. The dashed line is to guide the eye.

inclusion material respectively, and $\beta$ is the volume fraction of inclusions. The $n$ can be derived from the permittivity (Eq. 2), and the measured data agrees with the calculation results enabling deduction of the volume fraction of $\mathrm{TiO}_{2} \mathrm{NP}$ in each NPC film. Some deviations result from the formula assuming perfectlyuniform sphere-shaped NP whereas the actual NPC films contain non-uniform random-shaped NP. Since the NPC with $100 \%$ of the $\mathrm{TiO}_{2}$ weight ratio cannot be used for patterning due to the absence of monomer and photo-initiator, the maximum $n$ of 1.94 at the wavelength of $532 \mathrm{~nm}$ can be achieved near the weight ratio of $80 \%$ (Fig. 1e), and the corresponding volume fraction of $\mathrm{TiO}_{2}$ NPs is approximately $40 \%$. This is the condition used in this work.

Design and simulation of metalens. Rigorous coupled-wave analysis (RCWA) is used to simulate the optical properties of the NPC-based nanostructures with the measured $n$. Anisotropic rectangular nanorods induce geometric phase of the transmitted cross-polarized light by structure rotation. Broadband operation is a unique advantage of geometric phase due to its wavelength independence, so we use rectangular nanorods as the unit structure of the PPML (Fig. 2a). Cross-polarized light is affected by geometric phase whereas transmission of co-polarized light is irrelevant to structure rotation. Therefore, cross-polarization transmittance (CPT) is directly connected to the focusing efficiency of the PPML. When a left-handed circularly polarized
(LCP) beam is normally incident to the unit structure, the Jones vector of the outgoing wave can be written as ${ }^{33}$

$$
\frac{T_{\mathrm{L}}+T_{\mathrm{S}}}{2}\left[\begin{array}{l}
1 \\
i
\end{array}\right]+\frac{T_{\mathrm{L}}-T_{\mathrm{S}}}{2} \exp (i 2 \alpha)\left[\begin{array}{c}
1 \\
-i
\end{array}\right] \text {, }
$$

where $T_{\mathrm{L}}$ and $T_{\mathrm{S}}$ represent the complex transmission coefficients under light incidence of linear polarization along the long and short axis of the nanorod respectively, and $\alpha$ is the rotation angle of the nanorod along the $z$-axis. The CPT is the magnitude of the cross-polarization component (Eq. 3), and we calculate the CPT of the nanorod by changing its length from 100 to $400 \mathrm{~nm}$ and width from 50 to $200 \mathrm{~nm}$ (Fig. 2b). These ranges are determined considering fabrication compatibility. The designed unit structure yields higher CPT at short wavelengths in the visible; this high CPT is a result of the near-zero extinction coefficient of the NPC (Fig. 2c).

The higher $n$ of the NPC usually benefits from the higher CPT. We calculate the CPT of rectangular nanostructures based on the measured $n$ of the NPC. The whole relation between the CPT and $n$ is not simple at all, so we fix the structure height as $720 \mathrm{~nm}$ in the calculation to investigate local effects of the $n$ near the structural condition used in this work (Supplementary Fig. 1). As a result, we verify that the maximum CPT in the range of length and width is proportional to the weight ratio of $\mathrm{TiO}_{2} \mathrm{NPs}$ (Fig. 2d). 
a


b

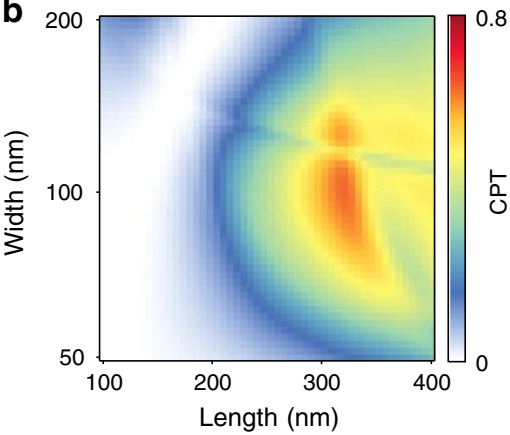

d

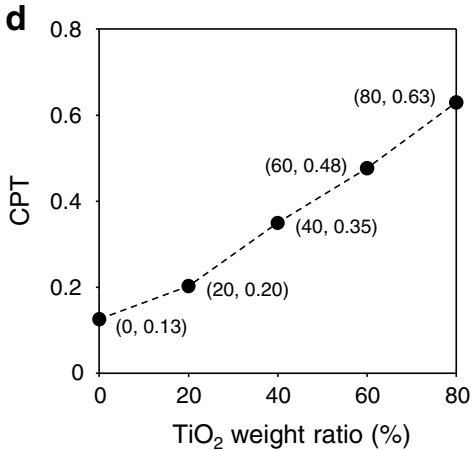

e



$30 \mu \mathrm{m}$

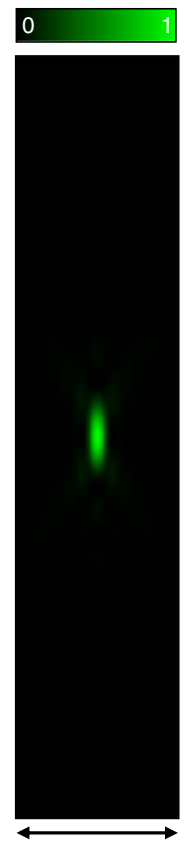

$30 \mu \mathrm{m}$

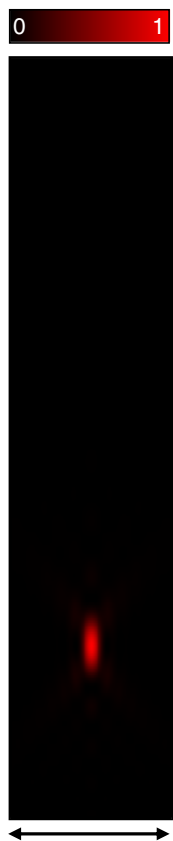

$30 \mu \mathrm{m}$

Fig. 2 Design and simulation of plum pudding metalens. a Configuration of unit structure consists of nanoparticle composite (NPC). Length: 330 nm; width: $100 \mathrm{~nm}$; height: $720 \mathrm{~nm}$; period: $450 \mathrm{~nm}$. Spheres inside the structure represent titanium dioxide $\left(\mathrm{TiO}_{2}\right)$ nanoparticles. b Calculation results of crosspolarization transmittance (CPT) of the NPC-based nanorod at the wavelength $\lambda=532 \mathrm{~nm}$. Height: $720 \mathrm{~nm}$; period: $450 \mathrm{~nm}$. c The calculated CPT of the unit structure. $\mathbf{d}$ The maximum CPT variation by the $\mathrm{TiO}_{2}$ weight ratio at $\lambda=532 \mathrm{~nm}$ when the height and period are fixed as 720 and $450 \mathrm{~nm}$, respectively. The dashed line is to guide the eye. e Optical field simulation of the metalens based on the Rayleigh-Sommerfeld diffraction formula with (left) $\lambda=450 \mathrm{~nm}$, (center) $\lambda=532 \mathrm{~nm}$, (right) $\lambda=635 \mathrm{~nm}$. The metalens is located at $z=0$, and designed to have $f_{\mathrm{d}}=2 \mathrm{~mm}$ at $\lambda_{\mathrm{d}}=532 \mathrm{~nm}$. The light passes through the metalens, then propagates along $+z$ direction. Each color represents a normalized field intensity in the $x z$ plane. Due to chromatic aberration, the designed phase gradient is not valid for other wavelengths resulting different focal lengths of $2.36 \mathrm{~mm}$ for $\lambda=450 \mathrm{~nm}$ and $1.67 \mathrm{~mm}$ for $\lambda=635 \mathrm{~nm}$.

The PPML is designed to follow a quadratic phase gradient to focus transmitted cross-polarized light without spherical aberration. The required phase gradient for the PPML is described as ${ }^{4}$

$$
\varphi(x, y)=\frac{2 \pi}{\lambda_{\mathrm{d}}}\left(f_{\mathrm{d}}-\sqrt{x^{2}+y^{2}+f_{\mathrm{d}}^{2}}\right),
$$

where $\lambda_{\mathrm{d}}=532 \mathrm{~nm}$ is the design wavelength, $f_{\mathrm{d}}=2 \mathrm{~mm}$ is the design focal length, $x$ and $y$ are coordinates of each nanorod. Each nanorod in the PPML requires $\alpha=\varphi / 2$ at each position (Eq. 3). The focusing characteristic can be simulated using the Rayleigh-Sommerfeld diffraction formula ${ }^{34}$ by discretizing the phase gradient by the sampling period $P=450 \mathrm{~nm}$ and the diameter $D=450 \mu \mathrm{m}$ of the PPML (Fig. 2e). At $\lambda=\lambda_{\mathrm{d}}$, transmitted cross-polarized light is focused at $f=f_{\mathrm{d}}$ as designed. Although the quadratic phase gradient is not valid for two other wavelengths of $\lambda=450$ and $635 \mathrm{~nm}$, they can be still focused, but at different positions resulting from chromatic aberration. The simulated focal lengths are $2.36 \mathrm{~mm}$ for $\lambda=450 \mathrm{~nm}$ and $1.67 \mathrm{~mm}$ for $\lambda=635 \mathrm{~nm}$. As $\lambda$ increases, the focal length of diffractive lenses decreases whereas that of a refractive lens increases ${ }^{35}$.

Fabrication of PPML. The PPML is replicated from the master mold using a single step of UV-NIL. EBL is used to fabricate the master mold ${ }^{36}$ (Fig. 3a), and it is coated by a self-assembled monolayer (SAM) that reduces the surface energy to facilitate mold release ${ }^{37}$. Hard polydimethylsiloxane (h-PDMS) is prepared by a typical method of mixing a vinyl polydimethylsiloxane (PDMS) prepolymer, modulator, hydrosilane prepolymer, platinum catalyst, and toluene ${ }^{38}$. Then, a stiff layer of $h$-PDMS is coated on the master mold covering the entire structure, and a flexible buffer layer of PDMS is coated on the $h$-PDMS layer (Fig. 3b). This composite soft-mold allows high-resolution pattern transfer due to the low viscosity and high stiffness of $h$ PDMS, which makes a soft-mold reusable. The compression modulus of the $h$-PDMS can widely vary from 0 to $10 \mathrm{~N} \mathrm{~mm}^{-2}$ depending on the vinyl ratio in prepolymer and hydrosilane ${ }^{39}$. The minimum feature size of the $h$-PDMS with the compression modulus of $9.7 \mathrm{~N} \mathrm{~mm}^{-2}$ is as small as $80 \mathrm{~nm}$, whereas pure PDMS in which modulus is $2 \mathrm{~N} \mathrm{~mm}^{-2}$ is usually capable of patterning down to $400 \mathrm{~nm}$-sized structures. The SAM is also coated on the released soft-mold to facilitate mold release in succeeding processes ${ }^{40}$. The NPC is prepared by blending methyl isobutyl ketone (MIBK) dispersed by $30-\mathrm{nm} \mathrm{TiO}_{2} \mathrm{NPs}$ with a mixture of a monomer, photo-initiator and $\mathrm{MIBK}^{41}$. Two solutions use the same solvent, so they can be mixed without any solute precipitation. The NPC is dropped on the glass substrate, then covered with the soft-mold. UV illumination with appropriate pressure hardens the NPC in the form of the master mold. After the release of the soft-mold, the PPML is created on the substrate (Fig. 3c).

Our fabrication method features rapid and high-resolution patterning of high-aspect-ratio dielectric nanostructures by a single process with strong substrate compatibility. Once the master mold is prepared, a single step of UV-NIL is enough to fabricate the PPML on the substrate without any secondary operations. UV-NIL is a rapid process, and the soft-mold is reusable; therefore, the PPML is highly competitive to commercial plastic lenses by injection molding. Due to the high stiffness of the NPC after UV curing, the aspect ratio of transferred patterns exceeds 10:1 which is not easy to achieve using typical 

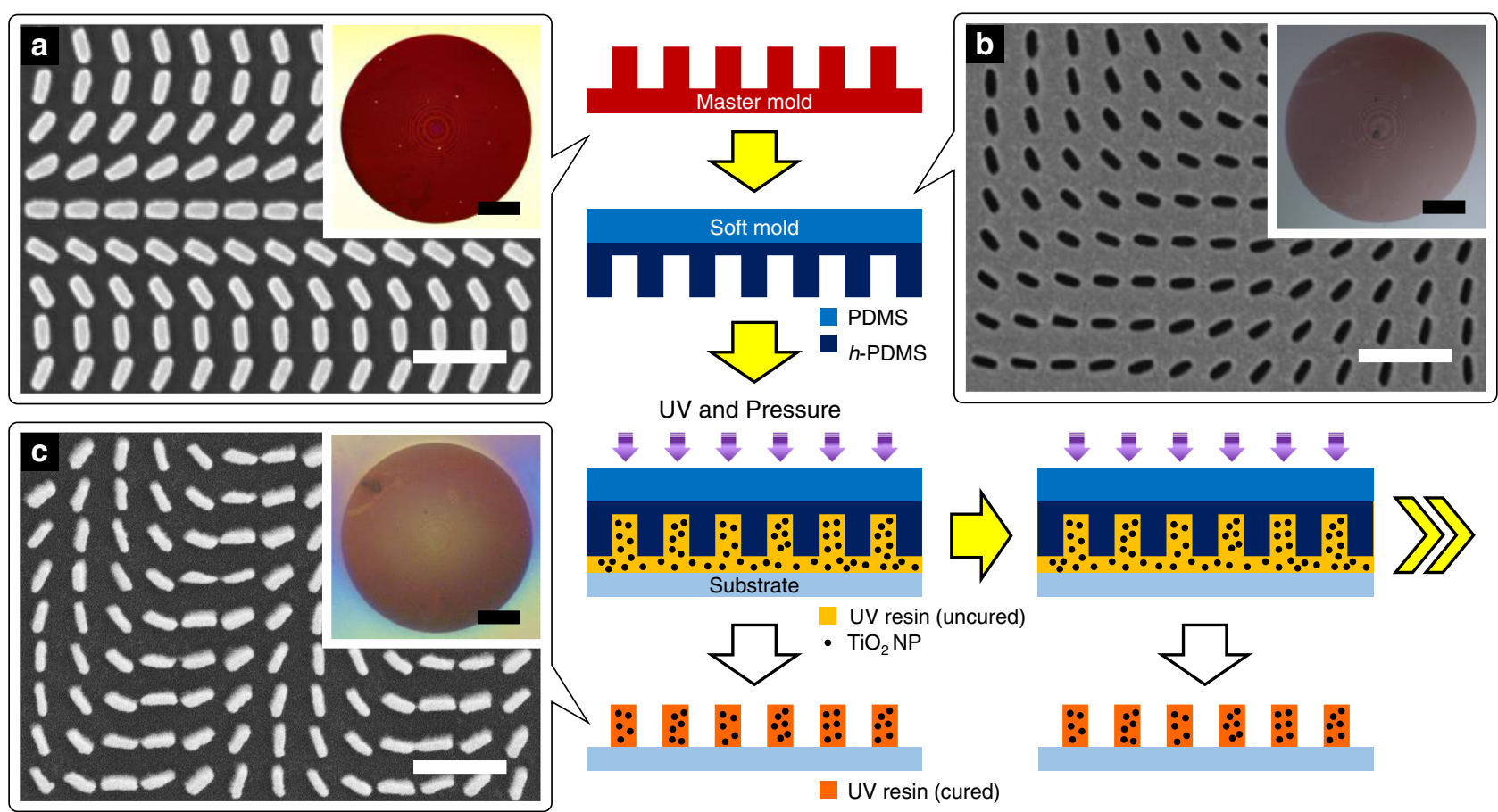

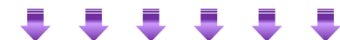



$$
\text { (1) }
$$

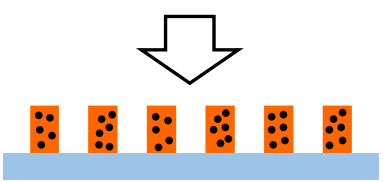

UV resin (cured)

Fig. 3 Fabrication schematic of plum pudding metalens. A master mold is fabricated by electron beam lithography and coated by the self-assembled monolayer (SAM) to improve mold release. Hard polydimethylsiloxane ( $h$-PDMS) is coated on the master mold, then a polydimethylsiloxane (PDMS) buffer layer is spread on the $h$-PDMS layer. A released soft-mold is also coated by the SAM to facilitate mold release in succeeding processes. Nanoparticle composite (NPC) which consists of titanium dioxide $\left(\mathrm{TiO}_{2}\right)$ nanoparticles (NPs) in a matrix of UV-curable resin is dropped on a glass substrate, and then it is covered by the soft-mold. UV light illumination with appropriate pressure hardens the NPC in the form of the master mold. The replication process is completed by releasing the reusable soft-mold from the substrate, and the soft-mold is reusable for additional replication. The final NPC patterns have a residual layer, but it is negligible because structures are much higher than the residual layer. a-c Scanning electron micrographs of the master mold, soft mold, and final plum pudding metalens, respectively. All scale bars: $1 \mu \mathrm{m}$. (insets) Optical micrographs of each. All scale bars: $100 \mu \mathrm{m}$.

sol-gel approaches ${ }^{42}$. The final NPC patterns have $20 \%$ shrinkage along each dimension regardless of the filling fraction of $\mathrm{TiO}_{2}$ NPs (Fig. 4), and the minimum linewidth of NPC patterns is approximately $100 \mathrm{~nm}$ (Fig. 5). The absence of secondary operations such as etching and annealing allows fabrication of the PPML on flexible substrates that are usually vulnerable to plasma and heat. Curved substrates are also compatible with our method due to the flexibility of the soft-mold. Therefore, our method is highly favorable for the fabrication of dielectric metasurfaces compared with conventional NIL (Supplementary Table 1).

Optical characterization of metalens. The focusing properties of the PPML are characterized using a customized optical setup (Fig. 6a). An LCP beam is normally incident to the PPML from the substrate to the structures. The iris tailors the incident beam to match the size of the PPML. The diverging beam behind the focal point is collected by a commercial objective lens of numerical aperture (NA) 0.7 , then passes through an analyzer that filters out the co-polarization component. Therefore, the camera receives only cross-polarized light that has right-handed circularly polarization (RCP) corresponding to a point spread function (PSF) of the PPML. The PSF for each wavelength is measured at each focal point (Fig. 6b). The focusing efficiency $\eta$ is defined as the ratio of optical power of the focal spot to the total incidence. Only the cross-polarized beam is focused at the focal point, so $\eta$ can be considered to be the CPT of unit structures. The measured $\eta$ of the PPML are $45 \%$ at $\lambda=450 \mathrm{~nm}, 33 \%$ at $\lambda=$ $532 \mathrm{~nm}$ and $11 \%$ at $\lambda=635 \mathrm{~nm}$. Due to fabrication defects, these are less than the calculated CPT, but the trends are similar.
Focal spots at each wavelength have the same full width at half maximum (FWHM) due to the low NA of the PPML. The measured focal lengths are $2.4 \mathrm{~mm}$ at $\lambda=450 \mathrm{~nm}, 2.0 \mathrm{~mm}$ at $\lambda=532 \mathrm{~nm}$ and $1.7 \mathrm{~mm}$ at $\lambda=635 \mathrm{~nm}$, which agree well with the theory of the focal length of diffractive lenses $f \approx f_{\mathrm{d}} \lambda_{\mathrm{d}} / \lambda$ where $\lambda$ represents a working wavelength ${ }^{35}$ (Fig. 6c). Considering $D=$ $450 \mu \mathrm{m}$, NAs of the PPML can be calculated as 0.09 at $\lambda=450 \mathrm{~nm}$, 0.11 at $\lambda=532 \mathrm{~nm}$ and 0.13 at $\lambda=635 \mathrm{~nm}$ by using the measured focal length for each wavelength. The measured FWHM of focal spots is $2.8 \mu \mathrm{m}$, which corresponds to a diffraction-limited $\mathrm{FWHM}^{4}$ of $\lambda /(2 \times \mathrm{NA})=2.4 \mu \mathrm{m}$ that is consistent for each wavelength due to the low NA (Fig. 6d). The diffraction-limited FWHM can also be derived from the Airy disk that represents the PSF formed by a perfect lens. The intensity of the airy disk is described as ${ }^{43}$

$$
I=I_{0}\left(\frac{2 \mathcal{J}_{1}\left(\frac{\pi D r}{\lambda f}\right)}{\frac{\pi D r}{\lambda f}}\right)^{2}
$$

where $I_{0}$ is the maximum intensity, $r$ is the radial distance from the center, and $J_{1}$ is the Bessel function of the first kind of order one.

The imaging characteristics of the PPML are characterized by using it as an objective lens (Fig. 6e). An LCP beam is normally incident to the target of the negative 1951 United States Air Force resolution test chart, and the transmitted beam from the target is collected by the PPML. This beam also passes through the analyzer to filter out the co-polarization component, so only the RCP beam reaches the camera. The imaging area includes 

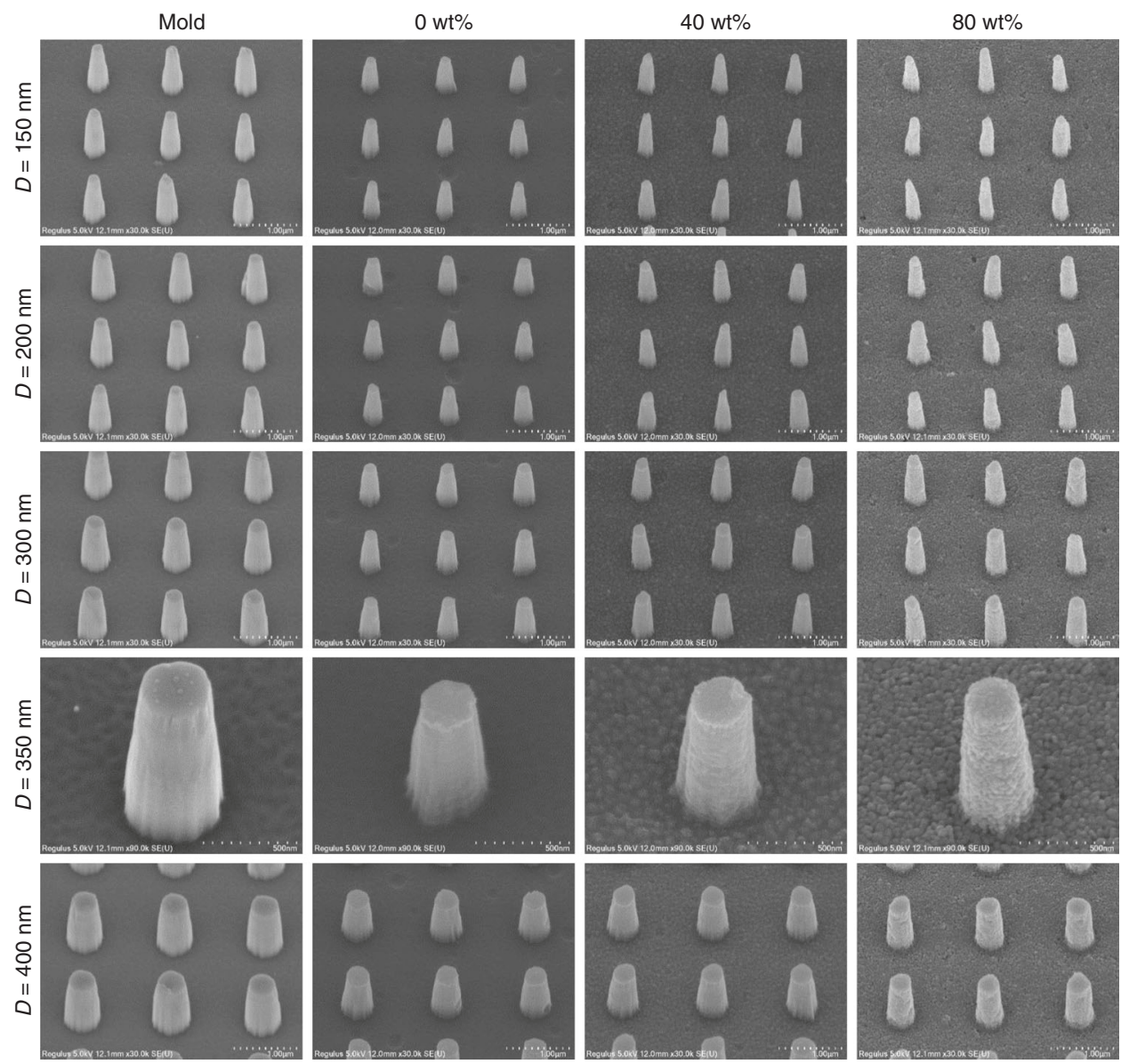

Fig. 4 Comparison of fabrication quality for circular nanopillar arrays. The titanium dioxide weight ratio varies from 0 to $80 \%$ while the diameter $D$ of circular nanopillars changes from 150 to $400 \mathrm{~nm}$. The height of nanopillars of the master mold is $1.2 \mu \mathrm{m}$, and all the patterns are tilted by $30^{\circ}$. It is worth noting that the electron beam of a scanning electron microscope deforms the nanoparticle composite patterns, i.e., bending and height shrinkage.

elements 5 and 6 of group 7 of the target; the field of view can be further enlarged by increasing the PPML size (Fig. 6f). The captured images show the same object with different magnifications by each wavelength due to the chromatic aberration of the PPML. Measured magnifications are proportional to the working wavelength and are therefore 0.84 at $\lambda=450 \mathrm{~nm}$ and 1.23 at $\lambda=$ $635 \mathrm{~nm}$, which are normalized to the magnification at $\lambda=532 \mathrm{~nm}$ (Fig. 6g). The positions of optical components behind the PPML are fixed, so the magnification is inversely proportional to the focal length of the PPML. The focal length is also inversely proportional to the working wavelength; therefore, the magnification is proportional to the working wavelength.

A modulation transfer function (MTF) provides information on the resolution and contrast that can be achieved by an optical imaging system. The MTF is defined as the magnitude of the Fourier transform of the square of an impulse response of the optical system normalized by a zero-frequency value. The impulse response of the optical imaging system corresponds to the PSF, so the MTF of our PPML-based imaging system can be described as $^{43}$

$$
\mathrm{MTF} \equiv\left|\frac{\iint I(x, y) \exp \left[-i 2 \pi\left(f_{\mathrm{x}} x+f_{\mathrm{y}} y\right)\right] d x d y}{\iint I(x, y) d x d y}\right|,
$$

where $I(x, y)$ denotes the PSF, $f_{\mathrm{x}}$ and $f_{\mathrm{y}}$ represent spatial frequencies along $x$-axis and $y$-axis, respectively. The cut-off frequency of our system reaches $400 \mathrm{~mm}^{-1}$ (Fig. 6h). This is much higher than the spatial frequency of the imaging target of $228 \mathrm{~mm}^{-1}$, so our system can distinguish each line pair of the target. The MTF of a diffraction-limited imaging system can be calculated by using the airy disk as the PSF due to the physical meaning of the Airy disk (Eq. 5). The FWHM of the PSF by the PPML is analogous to the airy disk. Therefore, the cut-off frequency of the diffraction-limited system is also analogous to our system whereas noise in the PSF by the PPML causes contrast reduction in our imaging system below the cut-off frequency compared with the diffraction-limited system.

\section{Discussion}

The experimental demonstration verifies the feasibility of the PPML as a practical low-cost metalens, and the current PPML can be further improved for future applications. The optical property of the NPC has a critical function in the PPML because the thickness of unit structures can be reduced by increasing the $n$ of the NPC. Our fabrication method can transfer structures that have high-aspect ratios, but reduction in structure thickness increases the reliability of the fabrication of the PPML. Mechanical stability of the current NPC can be further increased by using a hard material as the matrix. The material should be viscous to replicate the mold pattern but become mechanically stable after solidification by UV illumination or annealing. If the height of NPC structures reaches $1 \mu \mathrm{m}$ without any structural defects such as bending, the focusing efficiency of the PPML can exceed $85 \%$ at $\lambda=532 \mathrm{~nm}$ (Supplementary Fig. 2). Although 



Fig. 5 Comparison of fabrication quality for line arrays. The titanium dioxide weight ratio varies from 0 to $80 \%$ while the width $w$ of lines changes from 100 to $400 \mathrm{~nm}$. The height of line structures of the master mold is $1.2 \mu \mathrm{m}$, and all the patterns are tilted by $30^{\circ}$. It is worth noting that the electron beam of a scanning electron microscope deforms the nanoparticle composite patterns, i.e., bending and height shrinkage.

chromatic aberration is not corrected in this work, our method is also capable of realizing achromatic metalenses by stacking individual metalenses and phase correctors. It has been already reported that stacked metasurfaces including metalenses can improve imaging qualities in various ways such as coma aberration correction 44,45 , chromatic aberration correction in refractive optics $^{46}$, and multiwavelength achromatic focusing ${ }^{15}$. Given that the individual metasurfaces of these works can be manufactured at low cost by using our method, we believe this work can provide an experimental foundation for compound metalenses to fully compensate for the chromatic aberration.

In conclusion, we demonstrate a PPML, in which unit structures consist of NPCs to reduce fabrication costs. The NPC is composed of a UV-curable resin as a matrix that includes $\mathrm{TiO}_{2}$ NPs. The effective $n$ of the NPC is increased by the $\mathrm{TiO}_{2} \mathrm{NPs}$, so the NPC can be used directly as a material for metalenses. The mold pattern can be replicated by the NPC due to its UV-curable matrix. Therefore, the PPML can be fabricated with one step of NIL without any secondary operations such as thin-film deposition and etching, which decrease productivity and substrate compatibility. The fabricated PPML achieves a focusing efficiency of $33 \%$ at the target wavelength of $532 \mathrm{~nm}$, and the efficiency can be further increased by optimization of structure configuration. Focusing and imaging properties of the PPML are comparable to those of the diffraction-limited system. As a prototype of low-cost metalenses, the PPML will be a fundamental step toward commercialization of metalenses.

\section{Methods}

Fabrication of master mold. A 720-nm thick layer of hydrogenated amorphous silicon (a-Si:H) was deposited on a fused silica substrate by plasma-enhanced chemical vapor deposition. The electron beam resist (495 PMMA A2, Micro chem) was spin-coated on the sample at $2000 \mathrm{rpm}$, then baked at $180^{\circ} \mathrm{C}$ for $5 \mathrm{~min}$. A conductive polymer (Espacer 300Z, Showa denko) was spin-coated on the resist at $2000 \mathrm{rpm}$ to prevent charge accumulation. The pattern of the PPML was drawn on the resist by EBL (ELS-7800, Elionix). The sample was immersed in DI water for $1 \mathrm{~min}$ to remove the conductive polymer, then immersed in developing solution (MIBK:IPA $=1: 3$, Micro chem) for $12 \mathrm{~min}$ at $0{ }^{\circ} \mathrm{C}$. A 30 -nm thick chromium $(\mathrm{Cr})$ layer is deposited on the sample by electron beam evaporation. A lift-off process was conducted in acetone to define a Cr mask on the sample. Then, the a-Si:H thin film was etched along the $\mathrm{Cr}$ mask using inductively-coupled plasma reactive ion etching. The master mold was completed after removal of a $\mathrm{Cr}$ mask residue using Cr etchant.

Synthesis of NPC. The NPC was prepared by mixing two solutions. One is a mixture of a monomer (Dipentaerythritol penta-/hexa-acrylate, Sigma-aldrich), photo-initiator (1-Hydroxycyclohexyl phenyl ketone, Sigma-aldrich) and MIBK, and the other is $\mathrm{TiO}_{2}$ NP-dispersed MIBK (DT-TIOA-30MIBK(N30), Ditto technology). The mixing ratio of two solutions was controlled to achieve a weight ratio of $4 \mathrm{wt} \%$ for $\mathrm{TiO}_{2} \mathrm{NPs}, 0.7 \mathrm{wt} \%$ for monomer and $0.3 \mathrm{wt} \%$ for photo-initiator in the NPC.

Replication process of PPML. The master mold was coated by liquid-phase SAM to improve mold release in a solution mixture of a 1000:1 volume ratio of hexane and (heptadecafluoro-1,1,2,2-tetra-hydodecyl)trichlorosilane (H5060.1, JSI silicone) for $10 \mathrm{~min}$. $h$-PDMS solution was prepared by mixing $3.4 \mathrm{~g}$ of vinylmethyl copolymers (VDT-731, Gelest), $18 \mu \mathrm{L}$ of platinum-catalyst (SIP6831.2, Gelest), 0.1 g of modulator (2,4,6,8-Tetramethyl-2,4,6,8-tetravinylcyclotetrasiloxane, Sigmaaldrich), $2 \mathrm{~g}$ of toluene and $1 \mathrm{~g}$ of siloxane-based silane reducing agent (HMS-301, Gelest). The $h$-PDMS was spin-coated on the master mold at $3000 \mathrm{rpm}$ for $50 \mathrm{~s}$, 


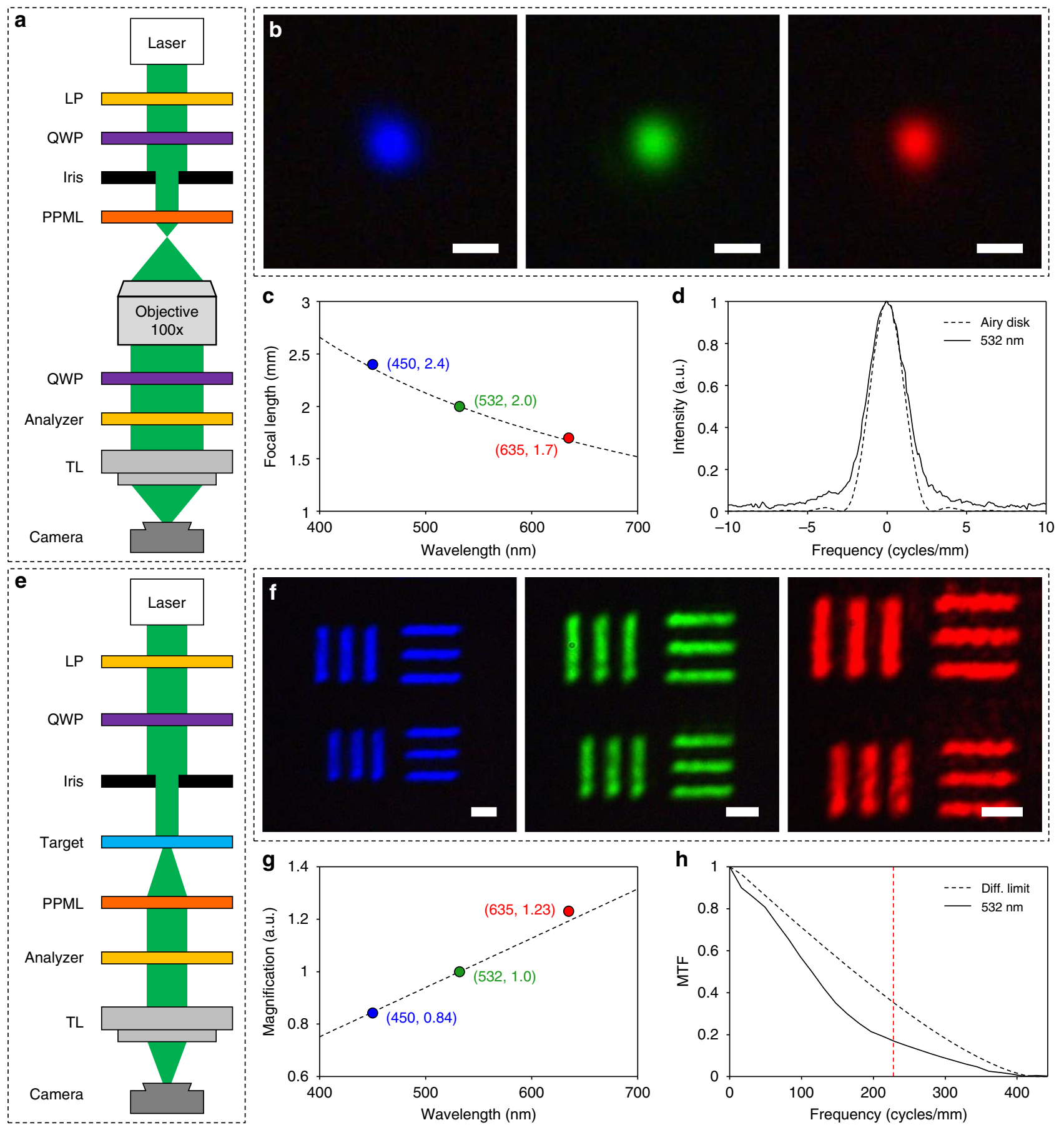

Fig. 6 Optical properties of plum pudding metalens. a Optical setup to characterize the focal spot. LP linear polarizer, QWP quarter-wave plate, TL tube lens, PPML plum pudding metalens. b Captured images of focal spots at wavelengths $\lambda=450,532$, and 635 , respectively. All scale bars: $5 \mu \mathrm{m}$. $\mathbf{c}$ Measured focal lengths denoted by three colored circles of $2.4 \mathrm{~mm}$ at $\lambda=450 \mathrm{~nm}, 2.0 \mathrm{~mm}$ at $\lambda=532 \mathrm{~nm}$ and $1.7 \mathrm{~mm}$ at $\lambda=635 \mathrm{~nm}$. Dashed line: theoretical prediction of the focal length $f=f_{\mathrm{d}} \lambda_{\mathrm{d}} / \lambda$ where $\lambda_{\mathrm{d}}$ and $f_{\mathrm{d}}$ represent the design wavelength $(532 \mathrm{~nm})$ and focal length $(2 \mathrm{~mm})$, respectively. d Comparison of focal spot intensity profiles at $\lambda=532 \mathrm{~nm}$. The full width at half maximum (FWHM) of the measured profile is $2.8 \mu \mathrm{m}$ that is closely consistent with the FWHM of the airy disk of $2.4 \mu \mathrm{m}$. e Optical setup to evaluate imaging properties of the PPML by using it as an objective lens. The analyzer allows only cross-polarized light to reach the camera. $\mathbf{f}$ Captured target images at $\lambda=450,532$, and $635 \mathrm{~nm}$, respectively. The imaging area includes the element 5 and 6 of group 7 in the negative 1951 United States Air Force resolution test chart. All scale bars: $5 \mu \mathrm{m}$. $\mathbf{g}$ Comparison of magnification by wavelengths. Dashed line: theoretical prediction of the relative magnification. Colored dots: measured image magnification at each wavelength. $\mathbf{h}$ Modulation transfer function (MTF) comparison between the PPML and a diffraction-limited imaging system. Red dashed line: location of the frequency value of $228 \mathrm{~mm}^{-1}$ that is the maximum spatial frequency of the target image.

then baked at $70^{\circ} \mathrm{C}$ for $2 \mathrm{~h}$. A degassed mixture of a 10:1 weight ratio of PDMS (Sylgard 184 A, Dow corning) and its curing agent (Sylgard 184 B, Dow corning) was poured on the $h$-PDMS layer, then baked at $100^{\circ} \mathrm{C}$ for $2 \mathrm{~h}$. The soft-mold was released from the master mold, then coated with vapor-phase SAM by a typical vaporizing process using (tridecafluoro-1,1,2,2-tetrahydrooctyl) trichlorosilane (SIT8174.0, Gelest) for $30 \mathrm{~min}$ at 5 Torr followed by DI water for $10 \mathrm{~min}$ at 10 Torr. The $3 \mu \mathrm{L}$ of NPC was dropped on a glass substrate, then it was covered by the soft-mold. UV light was illuminated for $5 \mathrm{~min}$ at 2 bar to harden the NPC (Nanosis 820, NND). The soft-mold was released to complete the replication process. 


\section{Data availability}

The data that support the findings of this study are available from the corresponding author upon reasonable request.

Received: 12 July 2019; Accepted: 17 April 2020;

Published online: 08 May 2020

\section{References}

1. $\mathrm{Yu}, \mathrm{N}$. et al. Light propagation with phase discontinuities: generalized laws of reflection and refraction. Science 334, 333-337 (2011).

2. Kim, I. et al. Outfitting next generation displays with optical metasurfaces. ACS Photonics 5, 3876-3895 (2018).

3. Staude, I. et al. Tailoring directional scattering through magnetic and electric resonances in subwavelength silicon nanodisks. ACS Nano 7, 7824-7832 (2013).

4. Khorasaninejad, M. et al. Metalenses at visible wavelengths: diffraction-limited focusing and subwavelength resolution imaging. Science 352, 1190-1194 (2016).

5. Yoon, G., Jang, J., Mun, J., Nam, K. T. \& Rho, J. Metasurface zone plate for light manipulation in vectorial regime. Commun. Phys. 2, 156 (2019).

6. Zheng, G. et al. Metasurface holograms reaching $80 \%$ efficiency. Nat. Nanotechnol. 10, 308-312 (2015).

7. Yoon, G., Lee, D., Nam, K. T. \& Rho, J. Pragmatic metasurface hologram at visible wavelength: the balance between diffraction efficiency and fabrication compatibility. ACS Photonics 5, 1643-1647 (2018).

8. Yoon, G. et al. Wavelength-decoupled geometric metasurfaces by arbitrary dispersion control. Commun. Phys. 2, 129 (2019).

9. Ni, X., Wong, Z. J., Mrejen, M., Wang, Y. \& Zhang, X. An ultrathin invisibility skin cloak for visible light. Science 349, 1310-1314 (2015).

10. Kumar, K. et al. Printing colour at the optical diffraction limit. Nat. Nanotechnol. 7, 557-561 (2012).

11. Jang, J. et al. Kerker-conditioned dynamic cryptographic nanoprints. $A d v$ Opt. Mater. 7, 1801070 (2019).

12. Lee, G.-Y. et al. Complete amplitude and phase control of light using broadband holographic metasurfaces. Nanoscale 10, 4237-4245 (2018)

13. Yoon, G., Lee, D., Nam, K. T. \& Rho, J. "Crypto-display" in dual-mode metasurfaces by simultaneous control of phase and spectral responses. ACS Nano 12, 6421-6428 (2018).

14. Avayu, O., Almeida, E., Prior, Y. \& Ellenbogen, T. Composite functional metasurfaces for multispectral achromatic optics. Nat. Commun. 8, 14992 (2017).

15. Zhou, Y. et al. Multilayer noninteracting dielectric metasurfaces for multiwavelength metaoptics. Nano Lett. 18, 7529-7537 (2018).

16. Chen, W. T. et al. Immersion meta-lenses at visible wavelengths for nanoscale imaging. Nano Lett. 17, 3188-3194 (2017).

17. Aieta, F. et al. Aberration-free ultrathin flat lenses and axicons at telecom wavelengths based on plasmonic metasurfaces. Nano Lett. 12, 4932-4936 (2012).

18. Huang, L. et al. Dispersionless phase discontinuities for controlling light propagation. Nano Lett. 12, 5750-5755 (2012).

19. Yoon, G., Lee, D., Nam, K. T. \& Rho, J. Geometric metasurface enabling polarization independent beam splitting. Sci. Rep. 8, 9468 (2018).

20. Lee, G.-Y. et al. Metasurface eyepiece for augmented reality. Nat. Commun. 9, 4562 (2018).

21. Khorasaninejad, M. et al. Polarization-insensitive metalenses at visible wavelengths. Nano Lett. 16, 7229-7234 (2016).

22. Chen, W. T. et al. A broadband achromatic metalens for focusing and imaging in the visible. Nat. Nanotechnol. 13, 220-226 (2018).

23. Wang, S. et al. A broadband achromatic metalens in the visible. Nat. Nanotechnol. 13, 227-232 (2018).

24. Shrestha, S., Overvig, A. C., Lu, M., Stein, A. \& Yu, N. Broadband achromatic dielectric metalenses. Light 7, 85 (2018).

25. Yoon, G., Kim, I. \& Rho, J. Challenges in fabrication towards realization of practical metamaterials. Microelectron. Eng. 163, 7-20 (2016).

26. $\mathrm{Wu}, \mathrm{W}$. et al. Midinfrared metamaterials fabricated by nanoimprint lithography. Appl. Phys. Lett. 90, 063107 (2007).

27. Chanda, D. et al. Large-area flexible $3 \mathrm{D}$ optical negative index metamaterial formed by nanotransfer printing. Nat. Nanotechnol. 6, 402-407 (2011).

28. Carlson, $\mathrm{K}$. et al. In vivo fiber-optic confocal reflectance microscope with an injection-molded plastic miniature objective lens. Appl. Opt. 44, 1792-1797 (2005).

29. Higashihara, T. \& Ueda, M. Recent progress in high refractive index polymers. Macromolecules 48, 1915-1929 (2015).
30. Pina-Hernandez, C. et al. A route for fabricating printable photonic devices with sub-10 nm resolution. Nanotechnology 24, 065301 (2013).

31. Werdehausen, D. et al. Design rules for customizable optical materials based on nanocomposites. Opt. Mater. Express 8, 3456-3469 (2018).

32. Humlicek, J. in Ellipsometry at the Nanoscale (eds Losurdo, M. \& Hingerl, K.) 145-178 (Springer, 2013)

33. Yu, N. \& Capasso, F. Flat optics with designer metasurfaces. Nat. Mater. 13, 139-150 (2014)

34. Shen, F. \& Wang, A. Fast-Fourier-transform based numerical integration method for the Rayleigh-Sommerfeld diffraction formula. Appl. Opt. 45, 1102-1110 (2006).

35. Aieta, F., Kats, M. A., Genevet, P. \& Capasso, F. Multiwavelength achromatic metasurfaces by dispersive phase compensation. Science 347, 1342-1345 (2015).

36. Yoon, G., Lee, D. \& Rho, J. Demonstration of equal-intensity beam generation by dielectric metasurfaces. J. Vis. Exp. 148, e59066 (2019).

37. Ulman, A. Formation and structure of self-assembled monolayers. Chem. Rev. 96, 1533-1554 (1996).

38. Odom, T. W., Love, J. C., Wolfe, D. B., Paul, K. E. \& Whitesides, G. M. Improved pattern transfer in soft lithography using composite stamps. Langmuir 18, 5314-5320 (2002)

39. Schmid, H. \& Michel, B. Siloxane polymers for high-resolution, high-accuracy soft lithography. Macromolecules 33, 3042-3049 (2000).

40. Jung, G.-Y. et al. Vapor-phase self-assembled monolayer for improved mold release in nanoimprint lithography. Langmuir 21, 1158-1161 (2005).

41. Choi, J.-H., Jo, H.-B., Choi, H.-J. \& Lee, H. Fabrication of $\mathrm{TiO}_{2}$ nano-tomicroscale structures using uv nanoimprint lithography. Nanotechnology 24, 195301 (2013).

42. Checcucci, S. et al. Multifunctional metasurfaces based on direct nanoimprint of titania sol-gel coatings. Adv. Opt. Mater. 7, 1801406 (2019).

43. Goodman, J. W. Introduction to Fourier Optics (Roberts and Company Publishers, 2005).

44. Arbabi, A. et al. Miniature optical planar camera based on a wide-angle metasurface doublet corrected for monochromatic aberrations. Nat. Commun. 7, 13682 (2016).

45. Groever, B., Chen, W. T. \& Capasso, F. Meta-lens doublet in the visible region. Nano Lett. 17, 4902-4907 (2017).

46. Chen, W. T. et al. Broadband achromatic metasurface-refractive optics. Nano Lett. 18, 7801-7808 (2018).

\section{Acknowledgements}

This work was financially supported by the National Research Foundation (NRF) grants (NRF-2019R1A2C3003129, CAMM-2019M3A6B3030637, NRF-2019R1A5A8080290, NRF-2018M3D1A1058997, and NRF-2015R1A5A1037668) funded by the Ministry of Science and ICT (MSIT) of the Korean government. H.L. acknowledges the NRF grant (NRF-2019K1A47A02113032) funded by MSIT and the Technology Innovation program (20000887 and N0002310) funded by the Ministry of Trade, Industry \& Energy (MOTIE) of the Korean government.

\section{Author contributions}

J.R. and H.L. conceived the idea and initiated the project. G.Y. designed the PPML and fabricated the master mold. K.K. and D.H. developed replication processes and replicated the PPML from the master mold. G.Y. did the optical characterization of the PPML. G.Y. J.R., and K.K. wrote the manuscript. J.R. guided the entire project. All authors participated in the discussion and confirmed the final manuscript.

\section{Competing interests}

The authors declare no competing interests.

\section{Additional information}

Supplementary information is available for this paper at https://doi.org/10.1038/s41467 020-16136-5.

Correspondence and requests for materials should be addressed to H.L. or J.R.

Peer review information Nature Communications thanks L. Jay Guo and the other, anonymous, reviewer(s) for their contribution to the peer review of this work. Peer reviewer reports are available.

Reprints and permission information is available at http://www.nature.com/reprints

Publisher's note Springer Nature remains neutral with regard to jurisdictional claims in published maps and institutional affiliations. 
(c) (i) Open Access This article is licensed under a Creative Commons Attribution 4.0 International License, which permits use, sharing, adaptation, distribution and reproduction in any medium or format, as long as you give appropriate credit to the original author(s) and the source, provide a link to the Creative Commons license, and indicate if changes were made. The images or other third party material in this article are included in the article's Creative Commons license, unless indicated otherwise in a credit line to the material. If material is not included in the article's Creative Commons license and your intended use is not permitted by statutory regulation or exceeds the permitted use, you will need to obtain permission directly from the copyright holder. To view a copy of this license, visit http://creativecommons.org/ licenses/by/4.0/.

(C) The Author(s) 2020 